Pacific Journal of Mathematic 


\section{COMPACT ELEMENTS OF WEIGHTED GROUP ALGEBRAS}

\section{F. GHAHRAMANI}

For a locally compact group $G$ let $L^{1}(G, \omega \lambda)$ be the weighted group algebra. We characterize elements $g \in L^{1}(G, \omega \lambda)$ for which the operator $T_{g}(f)=f * g\left(f \in L^{1}(G, \omega \lambda)\right)$ is compact. We conclude a result due to $S$. Sakai that if $G$ is a locally compact non-compact group, then 0 is the only compact element of $L^{1}(G, \lambda)$, and a result due to $\mathbf{C}$. Akemann that if $G$ is a compact group, then every element of $L^{1}(G, \lambda)$ is compact.

In a recent paper ([2], Theorem 2.2) W. G. Bade and H. G. Dales, among other things, characterize compact elements of $L^{1}\left(R^{+}, \omega\right)$. Niels Grønbæk, in his Ph.D. thesis ([6] Proposition 2.4), for a large class of semigroups (including cancellation semigroups) characterizes compact elements of the discrete weighted semigroup algebras.

In this paper, we characterize the compact elements of the weighted group algebras of locally compact groups. S. Sakai has proved that if $G$ is a locally compact non-compact group, then 0 is the only compact element of $L^{1}(G, \lambda)$, (see [10], Theorem 1), and C. Akemann has proved that if $G$ is a compact group, then every element of $L^{1}(G, \lambda)$ is compact ([1], Theorem 4). These two results will immediately follow from our characterization of the compact elements of the weighted group algebras. Also, a technique somewhat similar to ours provides other proofs for the Bade-Dales theorem (for bounded $\omega$ ) and the theorem of Gronbæk.

By a weight function on a locally compact group $G$ we mean a positive and continuous function $\omega$ on $G$ such that $\omega(s t) \leq \omega(s) \omega(t)$ $(s, t \in G)$. If $\lambda$ is a left Haar measure on $G$ and $\omega$ is a weight function on $G$, we set

$$
L^{1}(G, \omega \lambda)=\left\{f:\|f\|=\int_{G}|f(t)| \omega(t) d \lambda(t)<\infty\right\}
$$

Then, $L^{1}(G, \omega \lambda)$ is a Banach space: as usual, we equate functions equal $\lambda$ almost everywhere. Under the convolution product defined by the equation

$$
(f * g)(x)=\int_{G} f\left(x y^{-1}\right) g(y) d \lambda(y) \quad\left(f, g \in L^{1}(G, \omega \lambda)\right)
$$


$L^{1}(G, \omega \lambda)$ becomes a Banach algebra. We call an element $g \in L^{1}(G, \omega \lambda)$ compact if the operator $T_{g}(f)=f * g\left(f \in L^{1}(G, \omega \lambda)\right)$ is a compact operator.

Now we state the main result of this paper.

THEOREM 1. An element $g \in L^{1}(G, \omega \lambda)$ is compact if and only if the function $F_{g}$ defined on $G$ by

$$
F_{g}(s)=\int_{G} \frac{\omega(s t)}{\omega(s)}|g(t)| d \lambda(t) \quad(s \in G),
$$

vanishes at infinity.

Before we proceed to prove Theorem 1, we let $C_{0}(G, \omega)$ be the Banach space of all complex functions $f$ on $G$ such that $f / \omega \in C_{0}(G)$, and where the norm is taken to be

$$
\|f\|=\sup _{x \in G}\left|\frac{f(x)}{\omega(x)}\right| .
$$

Let $M(G, \omega)$ be the Banach space of all complex regular Borel measures $\mu$ on $G$ such that

$$
\|\mu\|=\int_{G} \omega(t) d|\mu|(t)<\infty
$$

then by the pairing $\langle\mu, \psi\rangle=\int_{G} \psi(x) d \mu(x)\left(\mu \in M(G, \omega), \psi \in C_{0}(G, \omega)\right)$ we have $\left(C_{0}(G, \omega)\right)^{*}=M(G, \omega)$ and we can define the product of $\mu$, $\nu \in M(G, \omega)$ by

$$
\int_{G} \psi(x) d(\mu * \nu)(x)=\int_{G} \int_{G} \psi(x y) d \mu(x) d \nu(y) \quad\left(\psi \in C_{0}(G, \omega)\right)
$$

to make $M(G, \omega)$ a Banach algebra. The map $f \rightarrow \mu_{f}$, where $d \mu_{f}(x)=$ $f(x) d \lambda(x)$ defines an isometric isomorphism from $L^{1}(G, \omega \lambda)$ into $M(G, \omega)$, and $L^{1}(G, \omega \lambda)$ can be identified with a closed ideal of $M(G, \omega)$. We define the topology (so) on $M(G, \omega)$ as follows: for a net $\left(\mu_{\alpha}\right) \subset$ $M(G, \omega)$ we let $\mu_{\alpha} \stackrel{(\text { so) }}{\rightarrow} \mu$ if and only if $\mu_{\alpha} * f \stackrel{\|\cdot\|}{\rightarrow} \mu * f$, for every $f \in$ $L^{1}(G, \omega \lambda)$, (see [4] and [5]). The algebra $L^{1}(G, \omega \lambda)$ possesses a bounded approximate left identity (see [8], p. 84).

The proof of the next lemma is formally the same as the proof of Theorem 20.4 of [7] and is therefore omitted. 
LEMma 1. The map $x \rightarrow 1 / \omega(x) \delta_{x}$ from $G$ into $M(G, \omega)$ is (so) continuous.

LEMMA 2. An element $g \in L^{1}(G, \omega \lambda)$ is compact if and only if it is a compact element of $M(G, \omega)$.

Proof. Suppose that the operator $T_{g}(f)=f * g\left(f \in L^{1}(G, \omega \lambda)\right)$ is compact. It is to be shown that the operator $\bar{T}_{g}(\mu)=\mu * g(\mu \in M(G, \omega))$ is compact.

Let $\left\{f_{\alpha}: \alpha \in A\right\}$ be a bounded approximate left identity for $L^{1}(G, \omega \lambda)$. If $\mu \in M(G, \omega)$, then

$$
\bar{T}_{g}(\mu)=\mu * g=\lim \mu * f_{\alpha} * g=\lim T_{g}\left(\mu * f_{\alpha}\right) .
$$

Hence the set $\left\{\bar{T}_{g}(\mu):\|\mu\| \leq 1\right\}$ is contained in the norm closure of the set $\left\{T_{g}\left(\mu * f_{\alpha}\right):\|\mu\| \leq 1, \alpha \in A\right\}$, which is compact by compactness of $T_{g}$. Thus, $\bar{T}_{g}$ is compact.

The converse is obvious, since $L^{1}(G, \omega \lambda)$ is a closed subspace of $M(G, \omega)$ and is invariant under $\bar{T}_{g}$ the restriction of which to $L^{1}(G, \omega \lambda)$ is $T_{g}$.

Proof of Theorem 1. Suppose $g$ is a compact element of $L^{1}(G, \omega \lambda)$, then by lemma 2 it is a compact element of $M(G, \omega)$. If the function $F_{g}$ defined by (1.1) does not vanish at infinity, then there exists an $\alpha>0$ such that for every compact set $K \subset G$, there exists $s \notin K$, with

$$
\left\|\bar{T}_{g}\left(\frac{1}{\omega(s)} \delta_{s}\right)\right\|=\int_{G} \frac{\omega(s t)}{\omega(s)}|g(t)| d \lambda(t) \geq \alpha .
$$

The set $\mathscr{K}$ of all compact subsets of $G$ is a directed set under set inclusion. For each $K \in \mathscr{K}$, choose $s(K) \notin K$ such that

$$
\left\|\bar{T}_{g}\left(\frac{1}{\omega(s(K))} \delta_{s(K)}\right)\right\|=\int_{G} \frac{\omega(s(K) t)}{\omega(s(K))}|g(t)| d \lambda(t) \geq \alpha .
$$

Thus, we obtain a net $\{s(K): K \in \mathscr{K}\}$ the terms of which satisfy (2). By the boundedness of the net

$$
\left\{\frac{1}{\omega(s(K))} \delta_{s(K)}: K \in \mathscr{K}\right\}
$$

and compactness of $\bar{T}_{g}$, there exists a subnet

$$
\left\{\frac{1}{\omega\left(s\left(K_{i}\right)\right)} \delta_{s\left(K_{i}\right)}: i \in I, \succ\right\}
$$


and a measure $\mu$ such that

$$
\bar{T}_{g}\left(\frac{1}{\omega\left(s\left(K_{l}\right)\right)} \delta_{s\left(K_{l}\right)}\right) \stackrel{\|\cdot\|}{\rightarrow} \mu .
$$

From (2) and (3) it follows that $\|\mu\| \geq \alpha$, whence there exists $\psi \in C_{0}(G, \omega)$ with $\|\psi\|=1$ and with

$$
|\langle\mu, \psi\rangle|>\alpha / 2
$$

By (3)

$$
\left|\left\langle\bar{T}_{g}\left(\frac{1}{\omega\left(s\left(K_{l}\right)\right)} \delta_{s\left(K_{\imath}\right)}\right), \psi\right\rangle\right| \rightarrow|\langle\mu, \psi\rangle| .
$$

From (4) and (5) it follows that there exist $i_{0} \in I$, such that for $i>i_{0}$, we have

$$
\left|\int_{G} \frac{\psi\left(s\left(K_{l}\right) y\right)}{\omega\left(s\left(K_{l}\right)\right)} g(y) d \lambda(y)\right|=\left|\left\langle\bar{T}_{g}\left(\frac{1}{\omega\left(s\left(K_{l}\right)\right)} \delta_{s\left(K_{l}\right)}\right), \psi\right\rangle\right|>\frac{\alpha}{2} .
$$

Choose $h \in L^{\mathrm{l}}(G, \omega \lambda)$ with compact support $K_{h}$ and such that $\|g-h\|<$ $\alpha / 4$. Then

$$
\begin{aligned}
\mid\left\langle\overline { T } _ { g } \left(\frac{1}{\omega\left(s\left(K_{l}\right)\right)}\right.\right. & \left.\left.\delta_{s\left(K_{l}\right)}\right), \psi\right\rangle-\left\langle\bar{T}_{h}\left(\frac{1}{\omega\left(s\left(K_{l}\right)\right)} \delta_{s\left(K_{l}\right)}\right), \psi\right\rangle \mid \\
& \leq \int_{G}\left|\frac{\psi\left(s\left(K_{i}\right) y\right)}{\omega\left(s\left(K_{l}\right) y\right)}\right| \frac{\omega\left(s\left(K_{i}\right) y\right)}{\omega\left(s\left(K_{l}\right)\right)}|g(y)-h(y)| d \lambda(y) \\
& \leq\|\psi\| \int_{G} \frac{\omega\left(s\left(K_{l}\right)\right) \omega(y)}{\omega\left(s\left(K_{i}\right)\right)}|g(y)-h(y)| d \lambda(y) \\
& =\|g-h\|<\frac{\alpha}{4} .
\end{aligned}
$$

Hence, for $i \succ i_{0}$, by (6) and (7), we have

$$
\begin{aligned}
\left|\left\langle\bar{T}_{h}\left(\frac{1}{\omega\left(s\left(K_{i}\right)\right)} \delta_{s\left(K_{t}\right)}\right), \psi\right\rangle\right| & >\left|\left\langle\bar{T}_{g}\left(\frac{1}{\omega\left(s\left(K_{i}\right)\right)} \delta_{s\left(K_{l}\right)}\right), \psi\right\rangle\right|-\frac{\alpha}{4} \\
& >\frac{\alpha}{2}-\frac{\alpha}{4}=\frac{\alpha}{4} .
\end{aligned}
$$

Now, let $K_{\psi} \in \mathscr{K}$, be such that

$$
\left|\frac{\psi(z)}{\omega(z)}\right|<\frac{\alpha}{4(1+\|h\|)}
$$


for $z \notin K_{\psi}$. Choose $i \succ i_{0}$ such that $K_{i} \supset K_{\psi} K_{h}^{-1}$. Then $s\left(K_{i}\right)$ satisfies (8). But, if $y \in K_{h}$, then $s\left(K_{i}\right) y \notin K_{\psi}$, whence

$$
\left|\frac{\psi\left(s\left(K_{i}\right) y\right)}{\omega\left(s\left(K_{i}\right) y\right)}\right|<\frac{\alpha}{4(1+\|h\|)} \text {. }
$$

Thus,

$$
\begin{aligned}
& \left|\left\langle\bar{T}_{h}\left(\frac{1}{\omega\left(s\left(K_{i}\right)\right)} \delta_{s\left(K_{i}\right)}\right), \psi\right\rangle\right| \\
& \quad=\left|\int_{K_{h}} \frac{\psi\left(s\left(K_{i}\right) y\right)}{\omega\left(s\left(K_{i}\right) y\right)} \frac{\omega\left(s\left(K_{i}\right) y\right)}{\omega\left(s\left(K_{i}\right)\right)} h(y) d \lambda(y)\right| \\
& \quad \leq \int_{K_{h}} \frac{\psi\left(s\left(K_{i}\right) y\right)}{\omega\left(s\left(K_{i}\right) y\right)}\left|\frac{\omega\left(s\left(K_{i}\right)\right) \omega(y)}{\omega\left(s\left(K_{i}\right)\right)}\right| h(y) \mid d \lambda(y) \\
& \quad \leq \frac{\alpha}{4(1+\|h\|)}\|h\| \leq \frac{\alpha}{4}
\end{aligned}
$$

which contradicts (8). Thus, $F_{g}$ vanishes at infinity.

Conversely, suppose that the function $F_{g}$ defined by (1.1) vanishes at infinity. If $g=0$, then it is obviously a compact element. If $g \neq 0$, then the vanishing of $F_{g}$ at infinity implies that $G$ is $\sigma$-compact. In fact, if for each positive integer $n$ we let $K_{n}$ be a compact subset of $G$ such that $\left|F_{g}(x)\right|<1 / n$ for $x \notin K_{n}$, then if $x \in G$, we have $F_{g}(x) \neq 0$, whence for some positive integer $n$ we have $1 / n<F_{g}(x)$. Hence $x \in K_{n}$, which implies $G=\cup_{n=1}^{\infty} K_{n}$. By Lemma 2 it suffices we prove that the operator $\bar{T}_{g}(\mu)=\mu * g(\mu \in M(G, \omega))$ is compact. The operator $\bar{T}_{g}$ is the adjoint of the operator $R_{g}$ defined on $C_{0}(G, \omega)$ by

$$
\left(R_{g} f\right)(x)=\int_{G} f(x y) g(y) d \lambda(y) \quad\left(f \in C_{0}(G, \omega), x \in G\right) .
$$

The map $\tau: C_{0}(G, \omega) \rightarrow C_{0}(G)$ defined by $(\tau f)(x)=f(x) / \omega(x)(x \in G)$ is a (linear) isometry. Therefore, it suffices to show that the operator $\tilde{R}_{g}=\tau R_{g} \tau^{-1}$, defined on $C_{0}(G)$ by

$$
\tilde{R}_{g}(f)(x)=\int_{G} \frac{\omega(x s)}{\omega(x)} f(x s) g(s) d \lambda(s) \quad\left(f \in C_{0}(G), x \in G\right),
$$

is compact.

Let $\left(f_{n}\right)$ be a bounded sequence in $C_{0}(G)$, and $\left(K_{n}\right)_{n=1}^{\infty}$ be as defined earlier. We note that $K_{n} \subset K_{n+1}, n=1,2, \ldots$ 
First we show that the sequence $\left(\tilde{R}_{g} f_{n}\right)$ has a subsequence the restriction of whose terms to $K_{1}$ converges uniformly to a function $h_{1} \in C\left(K_{1}\right)$. Let $s \in K_{1}$ and $\left(s_{\alpha}\right) \subset K_{1}$ be a net converging to $s$. Then,

$$
\begin{aligned}
& \left|\tilde{R}_{g} f_{n}\left(s_{\alpha}\right)-\tilde{R}_{g} f_{n}(s)\right| \\
& \quad=\left|\int_{G} f_{n}(y)\left(\left(\frac{1}{\omega(s)} \delta_{s} * g\right)(y)-\left(\frac{1}{\omega\left(s_{\alpha}\right)} \delta_{s_{\alpha}} * g\right)(y)\right) \omega(y) d \lambda(y)\right| \\
& \quad \leq \sup _{n}\left\|f_{n}\right\| \int_{G} \omega(y)\left|\left(\frac{1}{\omega(s)} \delta_{s} * g\right)(y)-\left(\frac{1}{\omega\left(s_{\alpha}\right)} \delta_{s_{\alpha}} * g\right)(y)\right| d \lambda(y) \\
& \quad=\sup _{s}\left\|f_{n}\right\|\left\|\left(\frac{1}{\omega\left(s_{\alpha}\right)} \delta_{s_{\alpha}}-\frac{1}{\omega(s)} \delta_{s}\right) * g\right\| \rightarrow 0,
\end{aligned}
$$

as $s_{\alpha} \rightarrow s$ uniformly in $\left(f_{n}\right)$, (by Lemma 1 ). Also the sequence $\left(\tilde{R}_{g} f_{n}\right)$ is uniformly bounded on $K_{1}$. Thus, by the Ascoli-Arzela theorem ([3], Theorem 7, p. 266) the set of restrictions of the terms of $\left(\tilde{R}_{g} f_{n}\right)$ to $K_{1}$ is a conditionally compact subset of $C\left(K_{1}\right)$, whence there exists a function $h_{1} \in C\left(K_{1}\right)$ and a subsequence $\left(\tilde{R}_{g} f_{1, k}\right)$ such that $\tilde{R}_{g} f_{1, k} \rightarrow h_{1}$, as $k \rightarrow \infty$, uniformly on $K_{1}$.

Let us now consider sequences $S_{1}, S_{2}, S_{3}, \ldots$, which we represent by the array

$$
\begin{aligned}
& S_{1}: \tilde{R}_{g} f_{1,1} \quad \tilde{R}_{g} f_{1,2} \quad \tilde{R}_{g} f_{1,3} \cdots . \\
& S_{2}: \tilde{R}_{g} f_{2,1} \quad \tilde{R}_{g} f_{2,2} \quad \tilde{R}_{g} f_{2,3} \cdots . \\
& S_{3}: \tilde{R}_{g} f_{3,1} \quad \tilde{R}_{g} f_{3,2} \quad \tilde{R}_{g} f_{3,3} \quad \tilde{R}_{g} f_{3,4} \cdots .
\end{aligned}
$$

and which have the following properties:

(a) $S_{n}$ is a subsequence of $S_{n-1}$, for $n=2,3,4, \ldots$.

(b) $\left(\tilde{R}_{g} f_{n, k}\right)$, when restricted to $K_{n}$, converges uniformly to a function $h_{n} \in C\left(K_{n}\right)$, as $k \rightarrow \infty$.

(c) The order in which the functions appear is the same in each sequence.

Thus, $h_{n+1}$ is an extension of $h_{n}$ from $K_{n}$ to $K_{n+1}$, and from the definition of $\tilde{R}_{g}\left(f_{n}\right)(x)$ we have

$$
\left|h_{n+1}(x)\right| \leq \sup _{m}\left\|f_{m}\right\| \frac{1}{n}, \quad \text { for } x \in\left(K_{n+1} \backslash K_{n}\right), n=1,2,3, \ldots
$$


Consider the diagonal array

$$
S: \tilde{R}_{g} f_{1,1} \quad \tilde{R}_{g} f_{2,2} \quad \tilde{R}_{g} f_{3,3} \cdots .
$$

By (c), the sequence $S$ (except possibly its first $n-1$ terms) is a subsequence of $S_{n}$ for $n=1,2, \ldots$. Hence (b) implies that $\left(\tilde{R}_{g} f_{n, n}\right)$ converges uniformly on $K_{i}$ to $h_{i} \in C\left(K_{i}\right), i=1,2, \ldots$. Now, if $h$ is a function on $G$, the restriction of which to $K_{i}$ is equal to $h_{i}$, then $\tilde{R}_{g} f_{n, n} \rightarrow h$, uniformly on $G$, and the proof is complete.

REMARK. Our method of finding the convergent subsequence $\left(\tilde{R}_{g} f_{n, n}\right)$ is similar to the well-known process of finding a pointwise convergent subsequence of a pointwise bounded sequence of functions defined on a countable set, (see [9], Theorem 7.23).

COROLLARY 1. If the group $G$ is a compact group, then every element of $L^{1}(G, \omega \lambda)$ is compact.

For the special case $\omega(t)=1(t \in G)$ we obtain:

COROLlaRY 2. (C. Akemann [1], Theorem 4.) If $G$ is a compact group, then every element of $L^{1}(G, \lambda)$ is compact.

Corollary 3. (S. Sakai [10], Theorem 1.) If $G$ is a locally compact non-compact group, then 0 is the only compact element of $L^{1}(G, \lambda)$.

Proof. If $g \neq 0$ is compact, then $\|g\|=\left|F_{g}(x)\right|<\frac{1}{2}\|g\|$ for every $x \notin K$, where $K$ is a proper compact subset of $G$, a contradiction.

Acknowledgements. The author wishes to thank Dr. H. G. Dales for a preprint of [2] and Dr. Niels Grønbæk for a copy of [6].

\section{REFERENCES}

[1] C. Akemann, Some mapping properties of the group algebras of a compact group, Pacific J. Math., 22 (1967), 1-8.

[2] W. G. Bade and H. G. Dales, Norms and ideals in radical convolution algebras, J. Functional Analysis, 41 (1981), 77-109.

[3] N. Dunford and J. Schwartz, Linear Operators, part 1, Interscience, New York, 1958.

[4] F. Ghahramani, Homomorphisms and derivations on weighted convolution algebras, J. London Math. Soc., (2) 21 (1980), 149-161.

[5] F. P. Greenleaf, Norm decreasing homomorphisms of group algebras, Pacific J. Math., 15, No. 4, (1965), 1187-1219. 
[6] N. Grønbæk, Derivations and semigroups, Commutative radical Banach algebras, Kobenhavens Universitet Matematisk Institut, Publikations Series, No. 3, 1980.

[7] E. Hewitt and K. A. Ross, Abstract Harmonic Analysis I, Springer-Verlag, Berlin, Heidelberg, New York, 1963.

[8] H. Rieter, Classical Harmonic Analysis and Locally Compact Groups, Oxford University Press 1968.

[9] W. Rudin, Principles of Mathematical Analysis, McGraw-Hill-Kogakusha, 1976.

[10] S. Sakai, Weakly compact operators on operator algebras, Pacific J. Math., 14 (1964), 659-664.

Received May 3, 1982 and in revised form February 22, 1983.

UNIVERSITY OF TEACHER EDUCATION

49 Mofateh Ave.

TEHRAN, IRAN 


\section{PACIFIC JOURNAL OF MATHEMATICS \\ EDITORS}

Donald BABBITT (Managing Editor)

University of California

Los Angeles, CA 90024

Hugo Rossi

University of Utah

Salt Lake City, UT 84112

C. C. Moore and Arthur Ogus

University of California

Berkeley, CA 94720
J. DugunduI

Department of Mathematics

University of Southern California

Los Angeles, CA 90089-1113

R. FinN and H. SAMELSON

Stanford University

Stanford, CA 94305

ASSOCIATE EDITORS

R. ARENS

E. F. BECKENBACH

B. H. NeUMANN

F. WOLF

K. YosHIDA (1906-1982)

\section{SUPPORTING INSTITUTIONS}

UNIVERSITY OF ARIZONA

UNIVERSITY OF BRITISH COLUMBIA

CALIFORNIA INSTITUTE OF TECHNOLOGY

UNIVERSITY OF CALIFORNIA

MONTANA STATE UNIVERSITY

UNIVERSITY OF NEVADA, RENO

NEW MEXICO STATE UNIVERSITY

OREGON STATE UNIVERSITY
UNIVERSITY OF OREGON

UNIVERSITY OF SOUTHERN CALIFORNIA

STANFORD UNIVERSITY

UNIVERSITY OF HAWAII

UNIVERSITY OF TOKYO

UNIVERSITY OF UTAH

WASHINGTON STATE UNIVERSITY

UNIVERSITY OF WASHINGTON 


\section{Pacific Journal of Mathematics}

\section{Vol. 113, No. $1 \quad$ March, 1984}

Flavio E. A. da Silveira, Rational homotopy theory of fibrations $\ldots \ldots \ldots \ldots 1$

Donald M. Davis, Desuspensions of stunted projective spaces ............ 35

Lou van den Dries, Exponential rings, exponential polynomials and

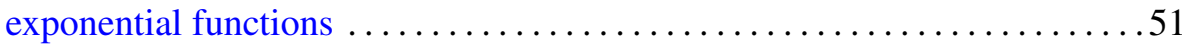

Fred Galvin and Samuel David Shore, Completeness in semimetric

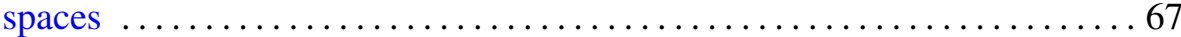

Fereidoun Ghahramani, Compact elements of weighted group algebras . . . 777

Munehiko Itōo, The closed image of a hereditary $M_{1}$-space is $M_{1} \ldots \ldots \ldots 85$

Elvira Laura Livorni, Classification of algebraic surfaces with sectional genus less than or equal to six. I. Rational surfaces .............. 93

H. Alan MacLean, Riesz sets and a theorem of Bochner ............ 115

E. Neher, Jordan triple systems with completely reducible derivation or

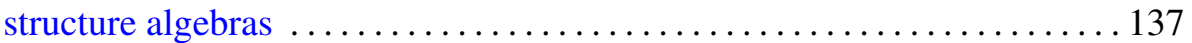

Joe Repka, Shalika's germs for $p$-adic GL( $n)$. I. The leading term $\ldots \ldots \ldots 165$

Joe Repka, Shalika's germs for $p$-adic GL( $n)$. II. The subregular term $\ldots \ldots 173$

Rae Michael Andrew Shortt, Borel density, the marginal problem and isomorphism types of analytic sets $\ldots \ldots \ldots \ldots \ldots \ldots \ldots \ldots \ldots \ldots \ldots \ldots \ldots$

Baruch Solel, The multiplicity functions of invariant subspaces for nonselfadjoint crossed products $\ldots \ldots \ldots \ldots \ldots \ldots \ldots \ldots \ldots \ldots \ldots \ldots . \ldots \ldots$

Su-win Yang, Self-intersection number of immersions and enumeration of nonstable vector bundles

W. M. Zajączkowski, Local solvability of nonstationary leakage problem for ideal incompressible fluid. II 